BBA 11059

\title{
MOLECULAR MOBILITIES AND THE LOWERED OSMOLALITY OF THE CHROMAFFIN GRANULE AQUEOUS PHASE
}

\author{
R. SEN and R.R. SHARP \\ Department of Chemistry, The University of Michigan, Ann Arbor, MI 48109 (U.S.A.)
}

(Received April 28th, 1982)

Key words: Chromaffin granule; NMR; Molecular mobility; Osmolality; Aqueous phase

Carbon-13 spin-lattice relaxation times, $T_{1}$, have been measured in whole adrenal medullary tissue slices, in suspensions of isolated chromaffin granules, in the reconcentrated chromaffin granule lysate, and in various model solutions containing catecholamines, ATP, chromogranins and $\mathrm{Ca}^{2+}$. Reorientational correlation times have been calculated at $10^{\circ} \mathrm{C}$ using $T_{1}$ data and nuclear $O$ verhauser enhancemments for protonated carbons on both catecholamines and nucleotides. Correlation times in all media are relatively short and characteristic of highly fluid aqueous phases. Adrenalin and ATP exhibit substantial differences in correlation times in all media, however, the ratio $\tau_{R}(\mathrm{ATP}): \tau_{R}$ (catecholamine) ranging from 2.4 in simple $3: 1$ adrenalin-ATP solutions to 4 in intact chromaffin granules. This difference, as well as the relatively high absolute reorientational mobilities of both components, confirms the importance of labile ionic interactions between ATP and catecholamines, but rules out the presence of high concentrations of base-stacked structures. Participation of the chromogranins in ternary complexes with catecholamines and ATP appears to be of minor importance. Ionic interactions to the protein are not reflected in either ${ }^{13} \mathrm{C} T_{1}$ values or chemical shifts of arginine or glutamate sidechain resonances, or in the ${ }^{13} \mathrm{C}$ chemical shifts of ATP or catecholamines. Very labile protein-ATP binding appears to be reflected in the correlation time measurements, however, which show selective immobilization of ATP relative to catecholamine in the presence of soluble protein. Osmotic measurements indicate that solutions containing adrenaline, ATP and $\mathrm{Ca}^{2+}$ are highly nonideal, but probably not sufficiently so to account fully for the osmotic stabilization of the chromaffin granule aqueous phase. Even in the absence of specific intermolecular complexation, the chromogranins, through their polyelectrolyte properties, exert a significant influence on the intragranular osmolality. The osmotic lowering due to polyion-counterion interactions has been estimated semiquantitatively using a theory developed by Oosawa.

\section{Introduction}

Chromaffin granules store high concentrations of catecholamines and ATP in an internal aqueous phase that is in osmotic equilibrium with the cytosol. The total concentration of low molecular weight solutes is approx. $730 \mathrm{mM}$, while the cytosolic osmolality is near 300 mosM. Recent NMR

Abbreviation: Hepes, $N$-2-hydroxyethylpiperazine- $N^{\prime}$-2-ethanesulphonic acid. studies [1] have shown that the internal aqueous phase contains all or nearly all of the stored catecholamines and adenine nucleotides as NMRvisible aqueous solutes. Evidently the internal medium is highly nonideal.

A number of suggestions have been offered to account for the lowered internal osmolality [2-20]. The most prominent of these is the hypothesis that catecholamines and ATP form aggregates of moderate to high molecular weight, possibly involving the mediation of divalent metal ions [3,5,6,9-15]. 
It has been suggested that base-stacking, reinforced by ionic interactions, plays a central role in stabilizing such structures. The presence of condensed catecholamine-ATP complexes has also been proposed $[5,9,10]$. The possible role of the soluble protein chromogranin in regulating the internal osmolality is not well understood. Isolated chromogranins exhibit very little binding capacity for catecholamines in equilibrium dialysis experiments $[17,18]$, but they reportedly bind a small fraction of the internal ATP very tightly [19]. The undialysable residue of the chromaffin granule lysate binds catecholamines and ATP by ion-exchange interactions at $\mathrm{pH} 5.6$ [20], which is wellestablished as the $\mathrm{pH}$ of resting chromaffin granules $[21,22]$. We have previously suggested that chromogranin, through its well-known properties as a largely random-coil polyelectrolyte, may significantly influence the osmotic coefficients of low molecular weight ions [7].

The present work expands a previous ${ }^{13} \mathrm{C}-\mathrm{NMR}$ study of molecular mobilities in chromaffin granules. Reorientational correlation times and ${ }^{13} \mathrm{C}$ chemical shifts of catecholamines and ATP have been measured in whole medullary tissue, in intact chromaffin granules isolated in $0.3 \mathrm{M}$ sucrose and in $0.145 \mathrm{M} \mathrm{K}_{2} \mathrm{SO}_{4}$, and in a series of model solutions containing mixtures of the soluble components. This kind of comparison gives a useful perspective for assessing the significance of intermolecular interactions in the chromaffin granule aqueous phase.

\section{Experimental}

Chromaffin granules were prepared from bovine adrenal medullary tissue by differential centrifugation following Kirshner's [23] procedure except that the isolation medium contained $0.3 \mathrm{M}$ sucrose $/ 10 \mathrm{mM}$ Hepes buffer $(\mathrm{pH}=7.0) / 0.5 \mathrm{mM}$ $\mathrm{Na}_{2} \cdot$ EDTA. Suspensions of the granule pellet $\left(0.3 \mathrm{~g}\right.$ pellet $/ \mathrm{ml}$ suspension) were prepared in ${ }^{2} \mathrm{H}_{2} \mathrm{O}$ solutions of $0.3 \mathrm{M}$ sucrose for use in the NMR experiments. Chromaffin granule suspensions free of sucrose, were obtained by washing once with 10 vol. of a $0.145 \mathrm{M} \mathrm{K}_{2} \mathrm{SO}_{4}$ solution in ${ }^{2} \mathrm{H}_{2} \mathrm{O}$ (330 mos $\mathrm{M}$ ) plus $10 \mathrm{mM}$ Hepes, $\mathrm{pH} 7.1$, and resuspending to a final concentration of $0.3 \mathrm{~g}$ pellet $/ \mathrm{ml}$ solution.
For experiments on whole medullary tissue, adrenals were obtained at a local abbatoir and placed on ice within $5 \mathrm{~min}$ of slaughter. Slices of medullary tissue were dissected within $45 \mathrm{~min}$ and placed in $0.2 \mathrm{ml}$ cold $0.145 \mathrm{M} \mathrm{K}_{2} \mathrm{SO}_{4}$ in ${ }^{2} \mathrm{H}_{2} \mathrm{O}$ in a $10 \mathrm{~mm}$ NMR tube. Tissue from a single gland sufficed to fill the tube. Spectra at $10^{\circ} \mathrm{C}$ were taken immediately, and the major lines were clearly visible after $20 \mathrm{~min}$. The spectrum of material maintained at $10^{\circ} \mathrm{C}$ changed very little over a $20 \mathrm{~h}$ period. In the $T_{1}$ experiments, 6000 scans $(1 \mathrm{~h})$ were accumulated at each $\tau_{1}$ value.

Preparation of the soluble lysate. Chromaffin granules pelleted in $0.3 \mathrm{M}$ sucrose as above were lysed by homogenization in 100 vol. double-distilled water. The membrane fraction was removed by two centrifugations at $35000 \times g$ for $20 \mathrm{~min}$ $\left(2^{\circ} \mathrm{C}\right)$. The supernatant was lyophilized and found to contain $36 \%$ by weight protein and $10 \%$ by weight catecholamines. The lyophilized residue was resuspended in ${ }^{2} \mathrm{H}_{2} \mathrm{O}$ to a protein concentration of $60 \mathrm{mg} / \mathrm{ml}$. The yellow-brown 'reconcentrated lysate' solution contained some aggregated material which was invisible in proton NMR spectra. The relative intensities of the well-resolved catecholamine and ATP peaks were not altered upon removing the solid by centrifugation.

Preparation of soluble protein. The reconcentrated lysate was dialyzed against several changes of $0.1 \mathrm{M} \mathrm{NH}_{4} \mathrm{Ac}(\mathrm{pH} 6.0)$ over $24 \mathrm{~h}$. The dialysate was lyophilized and stored at $-20^{\circ} \mathrm{C}$ until used. The residue was assayed and found to be $96 \%$ by weight protein.

Preparation of model solutions. A solution containing $0.55 \mathrm{M}$ adrenalin $/ 0.125 \mathrm{M}$ ATP $/ 120$ $\mathrm{mg} / \mathrm{ml}$ dialyzed protein was prepared by addition of adrenalin (free base) and ATP (monosodium salt) to a resuspension of the protein residue. The $\mathrm{p}^{2} \mathrm{H}$ was adjusted to 5.8 with ${ }^{2} \mathrm{HCl}$. The final solution contained a small amount of undissolved matter. Adrenalin and ATP used in all experiments were the free base and the monosodium salt supplied by Sigma Chemical Co. and Aldrich Chemical Co., respectively. These compounds were used without further purification after checking the composition by NMR and potentiometric titration. All solutions except that containing $\mathrm{Ca}^{2+}$ were $1 \mathrm{mM}$ in $\mathrm{Na}_{2}$. EDTA.

Protein assays were carried out on trichloro- 
acetic acid extracts by the method of Lowry et al. [24] with bovine serum albumin as the standards. Adrenalin assays were carried out using the trihydroxyindole method [25].

\section{NMR and osmotic measurements}

${ }^{1} \mathrm{H}$ decoupled ${ }^{13} \mathrm{C}-\mathrm{NMR}$ spectra were recorded on a JEOL-JNM-PFT/100 high resolution Fourier transform spectrometer. A spectral bandwidth of 5 $\mathrm{kHz}$ and 8192 data points gave a resolution of 1.22 $\mathrm{Hz}$ in all spectra. Peak integrations required for nuclear Overhauser enhancement measurements were carried out digitally with the EC-100 data system.

Spin-lattice relaxation times were measured by the inversion-recovery method $\left(180-\tau_{1}-90-\tau_{2}\right)_{n}$. Approximate $T_{1}$ values were estimated in preliminary measurements and used to insert an appropriate delay $\left(\tau_{2}>5 T_{1}\right)$ for recovery of the magnetization. Accumulations of 100-1000 scans at each $\tau_{1}$ value were used in measurements on the model solutions. 10000 scans with a $0.55 \mathrm{~s}$ delay were required with chromaffin granule suspensions, giving a signal-to-noise ratio of about $7: 1$ for the protonated adrenalin peaks in fully-relaxed spectra. $T_{1}$ values were obtained from semilogarithmic plots of peak amplitudes from between four and six $\tau_{1}$-values, in addition to three fully relaxed spectra.

Osmolalities at $0^{\circ} \mathrm{C}$ were measured using a Osmette A automatic osmometer (Precision Systems Incorporated).

\section{Data analysis}

Magnetic relaxation times of protonated ${ }^{13} \mathrm{C}$ nuclei provide a direct probe of the time scale of molecular reorientational mobility [7,26-28]. In general, the spin-lattice relaxation rate of a spin$1 / 2{ }^{13} \mathrm{C}$ nucleus can be written

$$
\begin{aligned}
\frac{1}{T_{1}}= & \left(\frac{1}{T_{1}}\right)_{\mathrm{dd}}+\left(\frac{1}{T_{1}}\right)_{\mathrm{dd} .0}+\left(\frac{1}{T_{1}}\right)_{\mathrm{inter}}+\left(\frac{1}{T_{1}}\right)_{\mathrm{p}} \\
& +\left(\frac{1}{T_{1}}\right)_{0}
\end{aligned}
$$

where

$$
\left(\frac{1}{T_{1}}\right)_{\mathrm{dd}}=\frac{N}{10} \frac{\hbar^{2} \gamma_{\mathrm{C}}^{2} \gamma_{\mathrm{H}}^{2}}{r_{\mathrm{CH}}^{6}}\left[\frac{\tau_{\mathrm{R}}}{1+\left(\omega_{\mathrm{H}}-\omega_{\mathrm{C}}\right)^{2} \tau_{\mathrm{R}}^{2}}\right.
$$

$$
\left.+\frac{3 \tau_{\mathrm{R}}}{1+\omega_{\mathrm{C}}^{2} \tau_{\mathrm{R}}^{2}}+\frac{6 \tau_{\mathrm{R}}}{1+\left(\omega_{\mathrm{H}}+\omega_{\mathrm{C}}\right)^{2} \tau_{\mathrm{R}}^{2}}\right]
$$

The first three terms arise from magnetic dipole coupling between the ${ }^{13} \mathrm{C}$ probe nucleus and (a) directly-bonded protons, (b) more distant protons in the same molecule, and (c) protons in other molecules. $\left(T_{1}\right)_{\mathrm{p}}^{-1}$ is a contribution from dissolved paramagnetic ions (if present) and $\left(T_{1}\right)_{0}^{-1}$ is a contribution from other relaxation pathways, most notably spin-rotation and chemical shift anisotropy. $\gamma_{C, H}$ and $\omega_{C, H}$ are the magnetogyric ratios and Larmor frequencies of the ${ }^{13} \mathrm{C}$ nucleus and the proton, $r_{\mathrm{C}-\mathrm{H}}$ is the directly-bonded ${ }^{13} \mathrm{C}-{ }^{1} \mathrm{H}$ bond distance, $N$ is the number of hydrogens directly bonded to the probe nucleus, $\tau_{R}$ is the reorientational correlation time of the carbon-hydrogen bond vector, and $2 \pi \hbar$ is Planck's constant. The motional information of $T_{1}$ measurements is contained in $\tau_{R}$, which is the average time required for a given carbon-hydrogen bond to reorient through approx. 1 radian. Reorientation may result from Brownian motion or from internal degrees of freedom, such as that associated with segmental motion of methylene groups or internal rotation of unhindered methyls. For protonated carbons, $\left(T_{1}^{-1}\right)_{\mathrm{dd}}$ normally dominates all the longer range dipolar interactions due to the $\left\langle r_{\mathrm{CH}}^{-6}\right\rangle$ factor in the dipolar coupling constant. Other diamagnetic relaxation mechanisms have been discussed elsewhere $[26,28]$ and for protonated carbons are expected to be negligible. The possible influence of paramagnetic ions is discussed further below.

In one of the systems studied (ATP in intact chromaffin granules), direct $T_{1}$ measurements were not practical because of low ${ }^{13} \mathrm{C}$ intensities. In this case, $\tau_{\mathrm{R}}$ was measured from the nuclear Overhauser enhancement. For a ${ }^{13} \mathrm{C}-{ }^{1} \mathrm{H}$ spin system in which the ${ }^{13} \mathrm{C} T_{1}$ is dominated by dipolar coupling to the proton (which again is normally true for protonated carbons), saturation of the ${ }^{1} \mathrm{H}$ spin levels with strong radio-frequency irradiation results in an enhanced steady-state magnetization for ${ }^{13} \mathrm{C}$. The ratio of enhanced to unenhanced intensities is the nuclear Overhauser enhancement (NOE), and is given by [29]

$\eta=1+\frac{\gamma_{H}}{\gamma_{C}}$ 
$\frac{\left(\frac{6}{1+\left(\omega_{H}+\omega_{C}\right)^{2} \tau_{R}^{2}}-\frac{1}{1+\left(\omega_{H}-\omega_{C}\right)^{2} \tau_{R}^{2}}\right)}{\left(\frac{1}{1+\left(\omega_{H}-\omega_{C}\right)^{2} \tau_{R}^{2}}+\frac{3}{1+\omega_{C}^{2} \tau_{R}^{2}}+\frac{6}{1+\left(\omega_{H}+\omega_{C}\right)^{2} \tau_{A}^{2}}\right)}$

where isotropic reorientation is assumed. The NOE is a known function of $\tau_{\mathrm{R}}$; a theoretical plot at $2.35 \mathrm{~T}$ is shown in Fig. 3. Overhauser enhancements provide a useful probe of molecular mobility over approximately an order of magnitude range of correlation times, which at $2.35 \mathrm{~T}$, is centered about $\tau_{R}=2$ ns.

\section{Results}

${ }^{13} \mathrm{C} T_{1}$ values have been measured and used to compute reorientational correlation times in slices of medullary tissue, in chromaffin granule suspensions, and in model solutions containing mixtures of the chromaffin granule aqueous solutes (Table I). Since reliable motional information is obtainable only from the $T_{1}$ values of protonated carbons, we have confined our attention to the C-2, C-5, C-6, $\mathrm{C}_{\alpha}$ and $\mathrm{C}_{\beta}$ resonances of catecholamines, and to the $\mathrm{C}-2, \mathrm{C}-8$, and $\mathrm{C}-1^{\prime}$ through $\mathrm{C}-5^{\prime}$ resonances of ATP. The methyl resonance of adrenalin was unsuitable for present purposes due to its relatively unhindered internal rotational motion. Peaks due to the protonated carbons of specific adenine and guanidine nucleotides are not resolved in spectra of intact chromaffin granules at $25.03 \mathrm{MHz}$, and the NMR data obtained from these spectra correspond to an average over in situ nucleotide populations.

Assignments of the catecholamine and nucleotide resonances observed in a suspension of chromaffin granules in $0.145 \mathrm{M} \mathrm{K}_{2} \mathrm{SO}_{4}\left(\mathrm{p}^{2} \mathrm{H}\right.$ 6.0) are shown in Fig. 1. The protonated ATP resonances are indicated by arrows. It is seen from the spectrum that C-8 (ATP) has unexpectedly low amplitude due to selective broadening. This feature has been noted previously in the reconcentrated lysate and shown to result very probably from free $\mathrm{Cu}^{2+}$, present at a total concentration near $200 \mu \mathrm{M}$. Exchangeable paramagnetic metal ions are evidently likewise present in the chromaffin granule aqueous phase and will in general provide a $T_{1}$ pathway for those nuclear resonances that are proximal to the metal binding site (the paramagnetic dipolar contribution to $T_{1}$ falls off as $\left.r^{-6}\right) . \mathrm{Cu}^{2+}$ binds to the phosphate esters of ATP and bridges to $\mathrm{N}-7$ on the adenine base. Thus, the ${ }^{13} \mathrm{C}$ linewidths and $\left(T_{1}^{-1}\right)$ values of four of the

\section{TABLE I}

$T_{1}$ VALUES (25.03 MHz) AND REORIENTATIONAL CORRELATION TIMES OF CATECHOLAMINES AND ATP IN WHOLE MEDULLARY TISSUE, IN ISOLATED CHROMAFFIN GRANULES, AND IN MODEL SOLUTIONS

Tabulated values are averaged over C-2, C-5 and C-6 for catecholamines and C-1, C-4, C-2, for ribose in ATP. All solutions are at pH 5.6, and solutions $1,2,3,5$ contain $1 \mathrm{mM}$ EDTA. Concentrations in the model solutions are: $0.55 \mathrm{M}$ adrenalin; $0.125 \mathrm{M}$ ATP; $30 \mathrm{mM}$ $\mathrm{Ca}^{2+} ; 120 \mathrm{mg} / \mathrm{ml}$ chromogranins.

\begin{tabular}{|c|c|c|c|c|c|}
\hline \multicolumn{2}{|l|}{ Sample } & \multicolumn{2}{|c|}{ Adrenalin } & \multicolumn{2}{|c|}{ ATP(average) } \\
\hline & & $N T_{1}(\mathrm{~s})$ & $\tau_{\mathrm{R}}(\mathrm{ns})$ & $N T_{1}(\mathrm{~s})$ & $\tau_{R}(\mathrm{~ns})$ \\
\hline \multirow[t]{2}{*}{ 1. Adrenalin } & $10^{\circ} \mathrm{C}$ & 0.464 & 0.096 & & \\
\hline & $25^{\circ} \mathrm{C}$ & 0.836 & 0.058 & & \\
\hline 2. ATP & $10^{\circ} \mathrm{C}$ & & & 0.132 & 0.365 \\
\hline \multirow[t]{2}{*}{ 3. Adrenalin + ATP } & $10^{\circ} \mathrm{C}$ & 0.228 & 0.20 & 0.104 & 0.48 \\
\hline & $30^{\circ} \mathrm{C}$ & 0.264 & 0.17 & & \\
\hline 4. Adrenalin + ATP $+\mathrm{Ca}^{2+}$ & $10^{\circ} \mathrm{C}$ & 0.182 & 0.25 & 0.077 & 0.68 \\
\hline 5.Adren. + ATP + chromogranins & $10^{\circ} \mathrm{C}$ & 0.171 & 0.27 & 0.076 & 0.70 \\
\hline 6. Lysate & $10^{\circ} \mathrm{C}$ & 0.125 & 0.38 & & \\
\hline \multicolumn{6}{|l|}{ 7. Chromaffin granules suspended in } \\
\hline $0.3 \mathrm{M}$ sucrose & $10^{\circ} \mathrm{C}$ & 0.100 & 0.50 & & \\
\hline $0.145 \mathrm{M} \mathrm{K}_{2} \mathrm{SO}_{4}$ & $5^{\circ} \mathrm{C}$ & 0.092 & 0.55 & & \\
\hline 8. Medullary tissue slices & $10^{\circ} \mathrm{C}$ & 0.110 & 0.45 & & \\
\hline
\end{tabular}




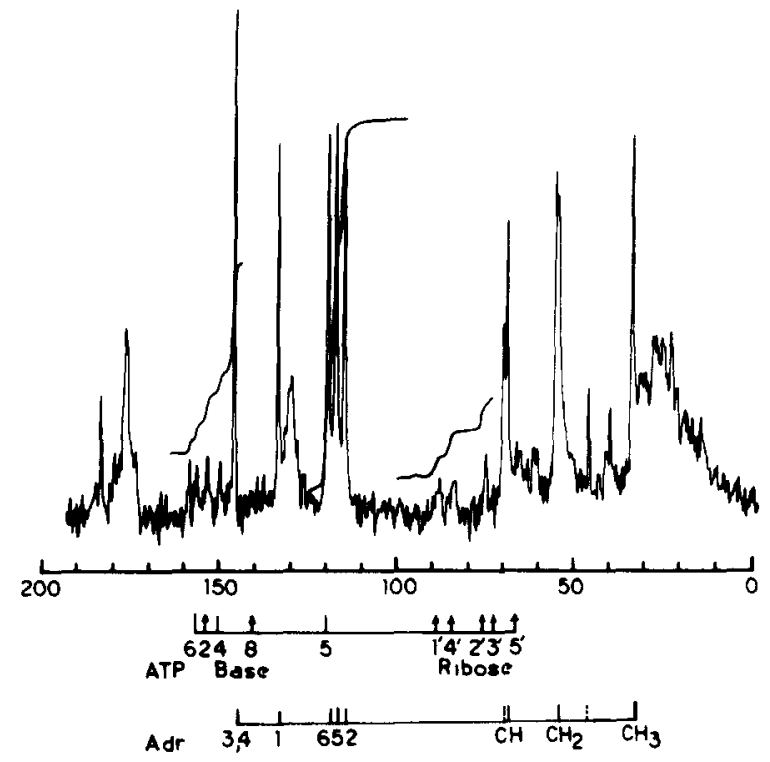

Fig. 1. ${ }^{13} \mathrm{C}$-NMR spectrum (25.03 MHz, 76000 transients) of chromaffin granules prepared by the method of Kirshner [23] and washed and resuspended in $0.145 \mathrm{M} \mathrm{K}_{2} \mathrm{SO}_{4}$. Chemical shift scale is ppm downfield of internal TMS assuming that the methyl carbon of adrenalin resonates at $34.2 \mathrm{ppm}$ [51]. Assignments of adrenalin, noradrenalin (dotted lines) and ATP are given. Aromatic resonances of adrenalin and noradrenalin are unresolved. Positions of protonated carbons of ATP are indicated by arrows.

adenine carbons contain a paramagnetic contribution that decreases in the order $\mathrm{C}-8>\mathrm{C}-5>\mathrm{C}-4>$ $\mathrm{C}-6$, but the resonances of $\mathrm{C}-2$ and the ribose carbons are unaffected by paramagnetic relaxation up to metal concentrations in the millimolar range $[30,31]$. For this reason, attention is focussed on these latter resonances in spectra of the lysate, chromaffin granules, and tissue slices. The model solutions contain $1 \mathrm{mM}$ EDTA, which effectively suppresses paramagnetic effects.

$T_{1}$ values of protonated ${ }^{13} \mathrm{C}$ resonances measure very directly $\tau_{\mathrm{R}}$ for the various ${ }^{13} \mathrm{C}-{ }^{1} \mathrm{H}$ bond vectors. To examine whether measured correlation times reflect segmental or internal motions as well as overall reorientation, the $T_{1}$ values of the various protonated carbons of adrenalin and of ATP were compared (Table II). Segmental or internal molecular motions will lengthen $T_{1}$ beyond the value corresponding to rigid reorientation and will lead to variation of $\tau_{R}$ values in a given molecule. After normalization for the number of directly bonded protons $(N), T_{1}$ values of the different protonated carbon resonances are reasonably constant (Table II), with the exception of the methyl carbon of adrenalin, which undergoes relatively free internal rotation, and C-5 (ATP), which is evidently the site of substantial segmental mobility. The relative constancy of $N T_{1}$ among the remaining ${ }^{13} \mathrm{C}$ resonances indicates that they provide a valid measure of overall molecular reorientational mobility. (As pointed out above, C-8 (ATP) is quite sensitive to paramagnetic metal ions and thus is not a reliable motional probe in the absence of EDTA.)

\section{Nuclear Overhauser enhancements}

Direct correlation time measurements using $T_{1}$ values of the ATP resonances were not feasible in intact chromaffin granules or in the reconcentrated lysate because of the prohibitively long accumulations required. Estimates of $\tau_{R}$ were made from nuclear Overhauser enhancements however, utilizing three unobscured protonated ribose reso-

\section{TABLE II}

${ }^{13} \mathrm{C}$ RELAXATION TIMES FOR ADRENALIN $\left(0.5 \mathrm{M} \mathrm{IN}{ }^{2} \mathrm{H}_{2} \mathrm{O}, 25^{\circ} \mathrm{C}\right.$, pH 5.6) AND Na ${ }_{3} \mathrm{ATP}\left(0.167 \mathrm{M}\right.$ in ${ }^{2} \mathrm{H}_{2} \mathrm{O}, 10^{\circ} \mathrm{C}$, pH 5.6$)$

$T_{1}$ values measured using the inversion-recovery method under broad-band decoupling conditions at $25.03 \mathrm{MHz}$.

\begin{tabular}{llll}
\hline Resonance & $N T_{1}(\mathrm{~s})$ & Resonance & $N T_{1}(\mathrm{~s})$ \\
\hline $\mathrm{C}-2$ & 0.832 & $\mathrm{C}-2$ & 0.117 \\
$\mathrm{C}-5$ & 0.844 & $\mathrm{C}-8$ & 0.127 \\
$\mathrm{C}-6$ & 0.832 & $\mathrm{C}-1^{\prime}$ & 0.151 \\
$\mathrm{C}-\alpha$ & 0.938 & $\mathrm{C}-2^{\prime}$ & 0.118 \\
$\mathrm{C}-\beta$ & 1.080 & $\mathrm{C}-3^{\prime}$ & 0.139 \\
$\mathrm{CH}$ & 1.54 & $\mathrm{C}-4^{\prime}$ & 0.141 \\
& & $\mathrm{C}-5^{\prime}$ & 0.194
\end{tabular}


TABLE III

RELATIVE INTENSITIES, NUCLEAR OVERHAUSER ENHANCEMENTS (NOE) AND REORIENTATIONAL CORRELATION TIMES OF NUCLEOTIDES IN CHROMAFFIN GRANULE SUSPENSIONS, IN LYSATE, AND IN MODEL SOLUTIONS.

Concentrations and $\mathrm{pH}$ values are given in Table I.

\begin{tabular}{|c|c|c|c|c|c|c|c|c|}
\hline \multirow[t]{3}{*}{ Sample } & & \multirow{2}{*}{$\begin{array}{l}\text { Catecholamine } \\
\text { aromatic } \\
\text { resonances }\end{array}$} & \multicolumn{2}{|c|}{ Nucleotide } & \multicolumn{2}{|c|}{ ' } & \multirow{2}{*}{\multicolumn{2}{|c|}{$\begin{array}{l}\tau_{\mathrm{R}}(\text { nucleotide })(\mathrm{ns}) \\
\text { from }\end{array}$}} \\
\hline & & & \multirow[t]{2}{*}{$\mathrm{C}-2$} & \multirow[t]{2}{*}{ C-1' } & \multirow[t]{2}{*}{$\mathrm{C}-2^{\prime}$} & \multirow[t]{2}{*}{ C-4' } & & \\
\hline & & & & & & & NOE & $T_{1}$ \\
\hline \multirow[t]{3}{*}{$\overline{1 .}$} & \multicolumn{6}{|c|}{ Adrenalin $+\mathrm{ATP}+\mathrm{Ca}^{2+}$} & 0.9 & 0.82 \\
\hline & INT & 1.00 & 0.69 & 0.95 & 0.82 & 0.86 & & \\
\hline & NOE & 2.97 & 2.06 & 2.84 & 2.45 & 2.57 & & \\
\hline \multirow[t]{3}{*}{2.} & \multicolumn{6}{|c|}{ Adrenalin + ATP + chromagranins } & 1.2 & 1.15 \\
\hline & INT & 1.00 & 0.82 & 0.78 & 0.70 & 0.86 & & \\
\hline & NOE & 2.94 & 2.45 & 2.34 & 2.10 & 2.57 & & \\
\hline \multirow[t]{3}{*}{3.} & Lysate & & & & & & 4.0 & - \\
\hline & INT & 1.00 & 0.51 & 0.44 & & & & \\
\hline & NOE & 2.92 & 1.54 & 1.32 & & & & \\
\hline \multirow[t]{4}{*}{4.} & Chromaffin gran & $\mathrm{s}$ in & & & & & & \\
\hline & $0.145 \mathrm{~K}_{2} \mathrm{SO}_{4}$ & & & & & & 2.0 & - \\
\hline & INT & 1.00 & 0.75 & 0.57 & - & 0.68 & & \\
\hline & NOE & 2.82 & 2.24 & 1.70 & - & 2.03 & & \\
\hline
\end{tabular}

nances, C-2', C-1' and C-4'. Digitally computed peak integrals are shown in the spectrum of Fig. 1, which is the result of 76000 transients accumulated over $12 \mathrm{~h}$ at $10^{\circ} \mathrm{C}$. The stability of chromaffin granule preparations in 357 mosM K ${ }_{2} \mathrm{SO}_{4}$ was monitored [1], and it was found that approx. $15 \%$ of the granules underwent lysis during this time period.

Table III lists intensities measured in protondecoupling experiments and the corresponding nuclear Overhauser enhancements calculated from Eqn. 3. To minimize the time required for spectral accumulation, enhancements were obtained from a comparison of intensities within a single spectrum accumulated during continuous proton decoupling using reference peaks (the aromatic catecholamine resonances) whose NOE is known by independent measurement. Referring back to Table III, it is seen that $\tau_{R}$ for the catecholamines ranges from 0.1 to $0.5 \mathrm{~ns}$ in the different systems studied, a range that corresponds to Overhauser enhancements between 2.98 and 2.85. Within experimental error, these enhancements equal the full theoretical value of 2.99. As a check on the measurement procedure, the enhancements of the catecholamine aromatic resonances were measured directly in a binary adrenalin-ATP solution using gated proton decoupling. The measured NOE of 2.88 is in good agreement with the independent measurement of $\tau_{\mathrm{R}}$ of $0.2 \mathrm{~ns}$ obtained from ${ }^{13} \mathrm{C} T_{1}$ values of adrenalin in the same solution. This kind of measurement verified that catecholamines have the full theoretical NOE in the systems studied.

Measured NOEs of ATP are plotted on a theoretical curve of Eqn. 3 in Fig. 2. There is good agreement between $\tau_{\mathrm{R}}$ determined from ${ }^{13} \mathrm{C} T_{1}$

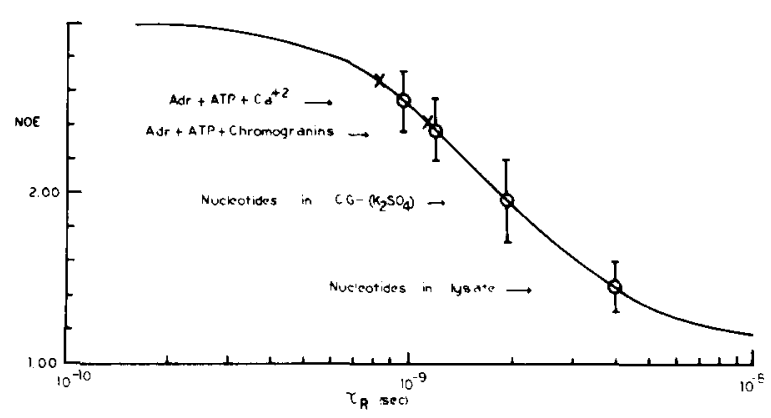

Fig. 2. Calculation of reorientational correlation times for nucleotides from nuclear Overhauser enhancements. 
values and from NOEs wherever crosschecks can be made (values derived from relaxation data are indicated by Xs in the Fig. 2). For the chromaffin granules and the reconcentrated lysate, where $T_{1}$ measurements on ATP were not feasible, the observed enhancement is intermediate between the limiting theoretical values, and thus provides a reasonably sensitive measure of the correlation time. These tabulated enhancements contain an estimated maximum uncertainty of $\pm 20 \%$ as indicated by the error bars in Fig. 2. The lysate appears to have a longer correlation time than do intact chromaffin granules, probably due to the presence of high concentrations of sucrose. Unfortunately, a similar measurement was not feasible in spectra of tissue slices since the ATP resonances in this case derive comparable intensity from intragranular and extragranular material.

\section{Trends in measured correlation times}

Fig. 3 contains a summary of measured correlation times: the values are averaged over several carbon resonances on either adrenalin or ATP as described above. In each of the eight systems studied, the catecholamines and ATP exhibit rotational mobilities characteristic of a highly fluid aqueous solution. The increase in rotational correlation time between a simple (adrenalin) $)_{3}$-ATP solution and whole tissue or intact chromaffin granules is only a factor of 2.5 for catecholamine

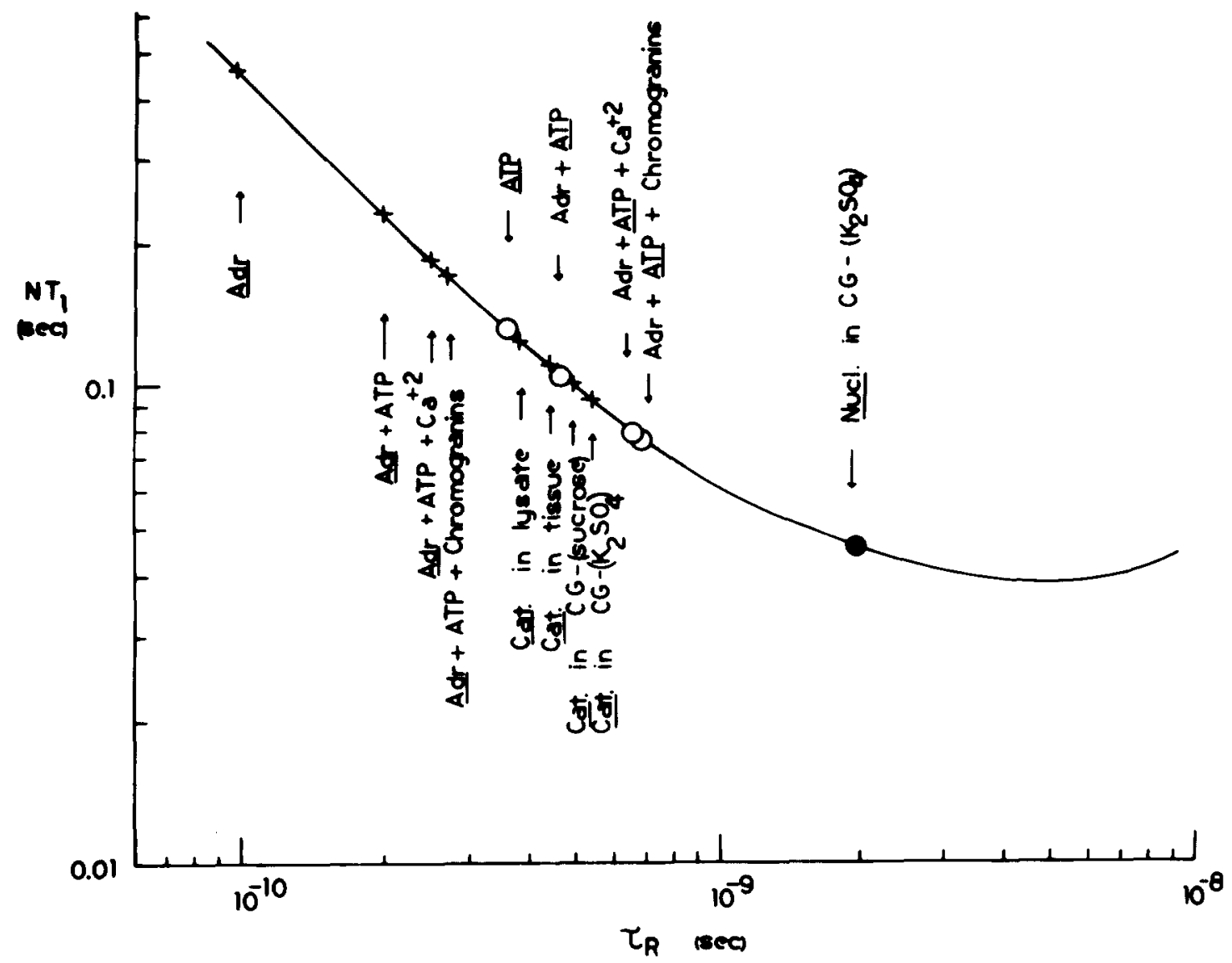

Fig. 3. Reorientational correlation times $\left(10^{\circ} \mathrm{C}\right)$ of catecholamines $(+)$ and ATP $(0)$ derived from the data in Table I using Eqn. 2 . The solid line is a theoretical plot of $N T_{1}$ vs. $\tau_{R}$ for protonated $C-13$ resonances at $25.03 \mathrm{MHz}$ assuming a $\mathrm{C}-\mathrm{H}$ bond length of 1.085 $\left(10^{-8}\right) \mathrm{cm}$. The $T_{1}$ values used are averages over $\mathrm{C}-2, \mathrm{C}-5$ and $\mathrm{C}-6$ aromatic resonances in the case of catecholamines and C-2, C-1', $\mathrm{C}-2^{\prime}, \mathrm{C}-3^{\prime}$ and $\mathrm{C}-4^{\prime}$ resonances in the case of ATP. The correlation time for nucleotides in chromaffin granules was calculated from NOE, rather than $T_{1}$ data, but is shown for comparison. 
and about 4 for nucleotides. Clearly a substantial portion of this difference, especially for the catecholamine, reflects an increase in viscosity.

The presence of binary adrenalin-ATP aggregation in the model solutions is indicated by the relatively large fractional increase in the correlation time of adrenalin that occurs upon addition of ATP. The observed $150 \%$ increase in $\tau_{R}$ is much greater than the viscosity change (20\%, unpublished results) and undoubtedly reflects a binary interaction as inferred in several previous studies $[2,3,5,11-15]$. The effect on $\tau_{\mathrm{R}}$ for ATP is much less than that on adrenalin. The ratio of correlation times, $\tau_{\mathrm{R}}(\mathrm{ATP}): \tau_{\mathrm{r}}$ (adrenalin), in the mixture is still farily large, about 2.4 , indicating that base portions of adrenaline and ATP reorient largely independently. Thus base-stacked structures, if present, are a minor component of the solution.

Addition of $30 \mathrm{mM} \mathrm{Ca}^{2+}$ to a binary adrenalinATP solution further lengthens the reorientational correlation times. Evidently $\mathrm{Ca}^{2+}$ stabilizes the catecholamine-nucleotide aggregates to a significant degree, which is in accord with measurements of the apparent molecular weight of the solute in similar solutions by sedimentation equilibrium techniques [33]. Possible structures have been suggested [14-16], but given the observed absolute difference of correlation times for adrenalin and the adenine resonances of ATP, it may be con- cluded that the aggregates are highly labile and that base-stacked structures are not dominant species in solution.

Addition of the soluble protein to an adrenalinATP solution to a final concentration of $120 \mathrm{mg}$. $\mathrm{ml}^{-1}$ lengthened the correlation times to a similar degree as was observed upon addition of $\mathrm{Ca}^{2+}$. In these solutions, the relative change of $\tau_{R}$, averaged over all resonances of ATP, is similar to the relative change of $\tau_{R}$ for the three protonated aromatic resonances of adrenalin.

In intact chromaffin granules (both isolated and in tissue slices), the correlation time increases to $0.45-0.55 \mathrm{~ns}$ for catecholamines and to $2.0 \mathrm{~ns}$ for ATP. Expressed as ratios relative to values in the adrenalin-ATP solution, $\tau_{R}$ of catecholamine in chromaffin granules has increased by a factor of 2.5 and $\tau_{R}$ of ATP by a factor of about 4 . Clearly the fluidity of the internal medium, as sensed on a microscopic level by the reorientational mobilities of these ions, is still fairly high. Absolute correlation times of nucleotide and catecholamine are quite different however, the ratio $\tau_{R}$ (ATP) : $\tau_{\mathrm{R}}$ (catecholamine) being 4.0 , thus emphasizing that these components reorient as independent units in the chromaffin granule aqueous phase. There appears to be little difference between intact chromaffin granules and medullary tissue slices as judged from the catecholamine and ATP reso-

Table IV

${ }^{13} \mathrm{C}$ CHEMICAL SHIFTS OF NUCLEOTIDE RESONANCES IN ADRENAL MEDULLARY TISSUE, ISOLATED CHRAFFIN GRANULES AND IN MODEL SOLUTIONS

Chemical shifts are measured in ppm downfield from methyl resonance of adrenalin. For resonance see Table II for numbering system. Sample compositions are given in Table I.

\begin{tabular}{|c|c|c|c|c|c|c|c|c|c|}
\hline \multirow[t]{2}{*}{ Sample } & \multicolumn{9}{|c|}{ Resonance } \\
\hline & C-6 & $\mathrm{C}-2$ & C-4 & C-8 & $C-1^{\prime}$ & $C-4^{\prime a}$ & $C-2^{\prime}$ & $-3^{\prime}$ & $C-5^{\prime a}$ \\
\hline 1. ATP & 121.25 & 118.94 & 115.39 & 107.00 & 54.42 & 50.90 & 41.80 & 37.48 & 32.63 \\
\hline 2. Adrenalin + ATP & 121.36 & 118.57 & 114.90 & 106.28 & 54.18 & 50.27 & 41.45 & 36.80 & 31.97 \\
\hline $\begin{array}{l}\text { 3. Adrenalin + ATP }+\mathrm{Ca}^{2+} \\
\text { 4. Adrenalin + ATP }\end{array}$ & 121.40 & 118.70 & 115.00 & 106.35 & 54.23 & 50.28 & 41.56 & 36.84 & 32.04 \\
\hline+ chrogranins & 121.60 & 118.50 & 114.8 & 106.4 & 54.41 & 50.30 & 41.55 & 36.92 & 32.04 \\
\hline $\begin{array}{l}\text { 5. Lysate } \\
\text { 6. Chraffin granules in }\end{array}$ & 121.5 & 118.7 & 115.05 & $106.4^{b}$ & 54.27 & - & 41.65 & - & - \\
\hline $0.145 \mathrm{M} \mathrm{K}_{2} \mathrm{SO}_{4}$ & 121.4 & 118.6 & 114.87 & $106.3^{b}$ & 54.33 & 50.36 & 41.36 & - & - \\
\hline 7. Medullary tissue & 121.2 & 118.6 & 114.98 & - & 54.21 & - & - & - & - \\
\hline
\end{tabular}

\footnotetext{
${ }^{a}$ Broadened due to unresolved ${ }^{31} \mathrm{P}-{ }^{13} \mathrm{C}$ coupling.

b Broadened from paramagnetic ion binding to phosphate ester.
} 
nances, although a direct determination of $\tau_{R}$ for intragranular ATP was not possible in tissue slices.

\section{Carbon-13 chemical shifts}

Chemical shifts in ${ }^{1} \mathrm{H}$ - and ${ }^{31} \mathrm{P}$-NMR spectra of chromaffin granules and of artificial mixtures of granular components have been reported $[14,15,35-37]$. The observation of upfield ${ }^{1} \mathrm{H}$ shifts has provided perhaps the main experimental support for the conclusion that base-stacked catecholamine-ATP aggregates occur in the intragranular milieu. ${ }^{13} \mathrm{C}$ chemical shifts that result from base stacking can be much larger than the corresponding proton shifts. Across the helix-coil transition of poly(A) for example, stacking of adenine bases produces ${ }^{13} \mathrm{C}$ shifts ranging from 0.8 to $2.7 \mathrm{ppm}$ at the adenine carbons and from 0.3 to $2.1 \mathrm{ppm}$ at the ribose carbons [38]. Thus ${ }^{13} \mathrm{C}$ chemical shifts should provide a relatively sensitive probe of rigid (e.g., base-stacked) aggregates.

Table IV contains a compilation of ${ }^{13} \mathrm{C}$ chemical shifts of nucleotides in medullary tissue, in isolated chromaffin granules, in the reconcentrated chromaffin granule lysate, and in various model solutions. Values are relative to the $\mathrm{CH}_{3}$ peak of adrenalin, which is the least perturbed adrenalin resonance with respect to concentration changes and ATP addition in earlier studies [15]. Large chemical shift changes of the order of 1-3 ppm are not observed. However, addition of adrenalin to an ATP solution does result in upfield chemical shifts of $0.4-0.7$ ppm for C-2, C-4 and C-8 on the adenine ring and $\mathrm{C}-4$ ' on the ribose moiety. The aromatic catecholamine resonances exhibit smaller $(<0.3 \mathrm{ppm})$ upfield shifts (data not shown). These results suggest that while hydrophobic interactions between aromatic rings may stabilize catecholamine-ATP aggregates to a minor degree, stacked structures are very labile and comprise at most about a third of the nucleotides; the same conclusion has been drawn much more directly from the $T_{1}$ data (see above).

The upfield shifts that are characteristic of ATP-catecholamine solutions are not greatly affected by addition of the soluble protein ( 120 $\mathrm{mg} / \mathrm{ml}$ ) and are also present in isolated chromaffin granules and in whole tissue. The close similarity of ${ }^{13} \mathrm{C}$ chemical shifts in intact chromaffin granules, in the protein-containing solutions and in binary catecholamine-ATP solutions suggests that binary aggregates are the main solute species in vivo and that ternary aggregates involving the soluble protein or $\mathrm{Ca}^{2+}$ are relatively unimportant.

The possibility that chromogranin participates in a ternary aggregate was investigated further by measuring ${ }^{13} \mathrm{C}$ chemical shifts and $T_{1}$ values of the well-resolved peaks from $\operatorname{Arg}\left(C_{\epsilon}\right)$ and Glu $\left(C_{\delta}\right)$. These two carbons might be expected to provide useful probes of binding since they are located close to the charge-bearing atom of one basic and one acidic amino acid residue. However, no significant shifts were observed in the ternary catecholamine-ATP-chromogranin solution relative to a solution of isolated chromogranins. Furthermore, relaxation measurements of these resonances in the reconcentrated lysate did not show anomalous shortening of $T_{1}$ relative to that of other sidechain resonances. Thus, the chromogranin C-13 resonances provided no evidence of selective immobilization of the charged sidechains, as might occur in an ionically-linked ternary aggregate.

\section{Osmotic measurements}

Osmolalties of solutions containing adrenalin, ATP and $\mathrm{Ca}^{2+}$ are shown in Fig. 4. Osmotic coef-

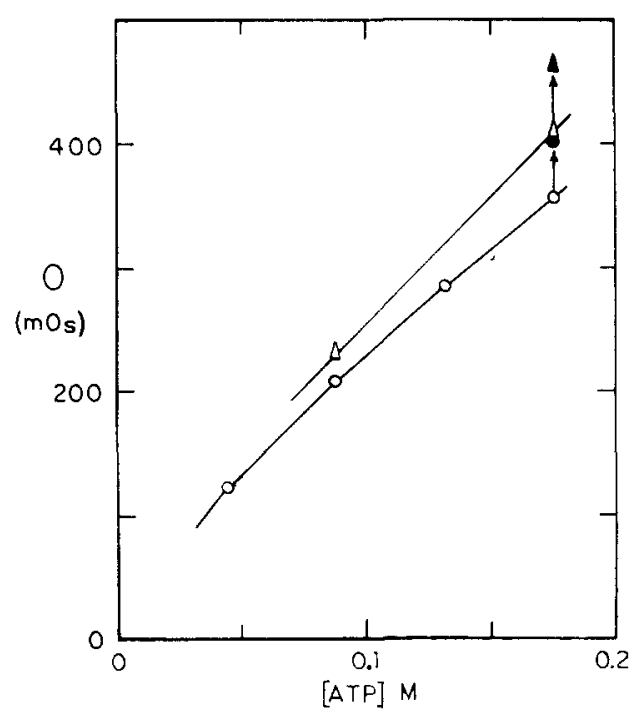

Fig. 4. Osmolalities of solutions containing adrenalin, ATP, $\mathrm{Ca}^{2+}, \mathrm{Na}^{+}$and $\mathrm{Cl}^{-}$at $0^{\circ} \mathrm{C}$. Solute compositions are $(\triangle)$ $\mathrm{Na}_{3}$ ATP, pH 5.56; (A) $\mathrm{Na}_{3} \mathrm{ATP}+30 \mathrm{mM} \mathrm{CaCl}_{2}, \mathrm{pH} 5.56$; (O) $\mathrm{Na}$ (Adr) ${ }_{2}$ ATP, pH 5.35; (O) $\mathrm{Na}$ (Adr) ${ }_{2} \mathrm{ATP}+30 \mathrm{mM}$ $\mathrm{CaCl}_{2}, \mathrm{pH}$ 5.35. 
ficients $\left(0^{\circ} \mathrm{C}\right)$ range between 0.51 for $0.176 \mathrm{M}$ $\mathrm{Na}$ (Adr) $)_{2}$ ATP and 0.586 for $0.176 \mathrm{M} \mathrm{Na}_{3}$ ATP, reflecting the high degree of nonideality that is expected for 3:1 electrolytes. Evidently (Adr) ${ }_{3}$ ATP solutions are sufficiently nonideal at $\mathrm{pH} 5.5$ to account for much of the osmotic stabilization of the chromaffin granule aqueous phase. It should be noted, however, that osmolalities at $37^{\circ} \mathrm{C}$ will be significantly higher than those measured at $0^{\circ} \mathrm{C}$. A $14 \%$ rise in osmolality will result from the increase in absolute temperature and a further rise will result from disaggregation. Thus, it appears that solution nonideality is the principal, but probably not the only, factor lowering osmotic coefficients in the chromaffin granule aqueous phase.

\section{Discussion}

Intermolecular associations among the soluble components of chromaffin granules

It is well-known that chromaffin granules are isoosmotic with the cytosol, for which the osmolality is of order 300 mosM, even though the total concentration of low molecular weight ions is at least $700 \mathrm{mM}[40,41]$. This fact implies either that the internal aqueous medium is highly nonideal, or else that a substantial portion of the catecholamines and nucleotides is sequestered in an osmotically inactive phase, such as a solid precipitate. We have investigated the latter possibility by using NMR integrals from proton spectra and found that most of the nucleotides and catecholamines are present in an NMR-visible form. The quantitation is less precise for nucleotides than for catecholamines, and it is possible that a significant fraction of the former (up to $20 \%$ ) could have escaped detection. Nevertheless, the total amount of NMR-invisible catecholamine and nucleotide consistent with chemical assay is clearly too small to account for the necessary osmotic lowering.

Berneis et al. [3,5] have observed a separation of two liquid phases in solutions of noradrenalin ATP and $\mathrm{Ca}^{2+}$ at a concentration and $\mathrm{pH}$ similar to that of the chromaffin granule interior. The denser phase is quite viscous, is highly concentrated in the soluble components, and contains aggregates of very high apparent molecular weight as measured by sedimentation equilibrium methods. This phase clearly has low osmolality, but its significance in chromaffin granules may be questioned since it does not form from adrenalin-containing solutions and since the upper consolute temperature is only about $7^{\circ} \mathrm{C}$. We have recorded NMR spectra of this phase and have observed ${ }^{1} \mathrm{H}$ linewidths far in excess of those in chromaffin granules or whole tissue [39], indicating that the phase is not present in vivo.

Evidently the bulk of catecholamines and nucleotides are present as relatively mobile, NMR-visible solutes in the aqueous phase, which is evidently a highly nonideal solution. Osmotic coefficients of model adrenalin-ATP solutions indicate that the weak interactions between the low molecular weight components account for much of the required nonideality (Fig. 4).

A number of studies have been directed toward the nature of the interactions between catecholamines, nucleotides and metal ions in recent years. There is no doubt that aggregates exist, and the ${ }^{13} \mathrm{C}$ relaxation data reported here confirm this fact. There also seems to be a consensus, based largely on NMR relaxation data and fluorescence lifetime measurements $[7,8,15]$ that these interactions are labile, and that the aggregates generally have low average molecular weight. On the other hand, there is less agreement concerning the extent to which ionic vs. hydrophobic interactions stabilize the aggregate. Our data are difficult to reconcile with the hypothesis of extensive base-stacking, since the average correlation times of catecholamines and of adenine ring of ATP differ substantially in all solutions studied; the ratio $\tau_{\mathrm{R}}(\mathrm{ATP}): \tau_{\mathrm{R}}$ (catecholamine) is about 4 in intact chromaffin granules. These and other observations $[2,8,15,44,45]$ concerning the lability of the aggregate suggest that the well-defined base-stacked structures that have been inferred from proton chemical shift studies $[12,13,14,16]$ are at most minor species in solution. Although upfield solution shifts have widely been interpreted as evidence of ring current effects in base-stacked structures, the empirical basis of this relation has been questioned [46] and is far from certain when small proton chemical shifts $(\delta \leqslant 0.1 \mathrm{ppm})$ are involved. Van der Waals shifts associated with changes in the solvating environment may easily exceed this magnitude.

We have found little direct evidence supporting 
participation of the protein in ternary catecholamine-ATP-protein complexes. Such interactions, if they exist, are not reflected in the ${ }^{13} \mathrm{C}$ chemical shift data or in the ${ }^{13} \mathrm{C} T_{1}$ values of arginine $(\mathrm{C}-\delta)$ or glutamate $(\mathrm{C}-\epsilon)$. It is noteworthy, however, that the reorientational mobility of ATP appears to be lowered differentially relative to that of catecholamines in the presence of protein (specifically, the ratio $\tau_{R}($ cat $): \tau_{R}($ ATP) increases from 2.4 for ATP-catecholamine solutions to 4 for the chromaffin granule interior (Fig. 3). The selective immobilization of nucleotide could reflect weak interactions, possibly both hydrophobic and ionic in nature, between nucleotides and soluble protein.

The polyelectrolyte properties of chromogranin and osmotic lowering in the chromaffin granule aqueous phase

Even in the absence of specific intermolecular complexation involving protein, the chromogranins, through their polyelectrolyte properties, can exert a significant influence on the intragranular osmolality. No definite function has been assigned to these proteins, which are present in very high concentration (approx. $170 \mathrm{mg} / \mathrm{ml}$ ) in the aqueous phase. Chromogranin $\mathrm{A}$ is largely a random coil peptide $[18,47,48,14,15]$ with an unusually large proportion of both anionic and cationic residues. We have previously suggested [7] that the polyelectrolyte properties of chromogranin, or possibly of a chromogranin-ATP aggregate, may have a significant effect on the osmotic properties of the internal medium.

The effect of a random-coil polyion (chromogranin) on the osmotic coefficient of a mobile counterion (catecholamine) may be viewed in a simplified manner as follows. The polyion, which bears a net number of charges, $N_{\mathrm{p}}$, occupies a domain in which the density of fixed charges is $\rho_{\mathrm{p}}$. Mobile counterions are distributed in solution in accord with the Poisson-Boltzmann equation,

$$
\nabla^{2} \psi=\frac{4 \pi}{\epsilon}\left(\rho_{\mathrm{p}}+e c^{+}\right)
$$

where $\psi$ is the electrical potential, $e$ is the protonic charge, $\epsilon$ is the dielectric constant and $c^{+}$is the local concentration of mobile counterions. The equilibrium distribution of counterions is such that it counteracts the buildup of large electrical potential throughout the polyion domain. In this way counterions may be 'bound' electrostatically and constrained to migrate with the polyion. The bound fraction is essentially osmotically inactive [49]. Inside the polyion domain, counterions retain the rotational and translational mobility characteristic of low molecular weight species, and thus their NMR spectra would be little perturbed from the solution state. This model reconciles at least qualitatively, the high reorientational mobilities of catecholamines and ATP in intact chromaffin granules with the low osmolality of the internal aqueous phase. It also provides a natural function for the high concentration of random-coil polyelectrolyte known to be present in chromaffin granules. We have explored this hypothesis semiquantitatively elsewhere [43]. The results are summarized in Fig. 5, where the osmotic coefficient $g^{\mathrm{c}}$ of catecholamines is plotted as a function of the number of net fixed charges $N_{\mathrm{p}}$ per $77000 \mathrm{Da}$ unit of chromogranin.

From the known amino acid composition of the chromogranins [40], $N_{\mathrm{p}}$ is $37 e^{-}$(this value assumes protonated histidines and takes account of charges on sialic acid). A fraction of mobile cations ( $\left.F_{\text {catecholamines }}\right)$ is required as counterion for the fixed charge and has lowered osmotic activity. The average $g^{\mathfrak{c}}$ for catecholamines in a medium of composition similar to that of the intragranular matrix has been calculated, following Oosawa [49], as a function of $N_{\mathrm{p}}$ and of volume fraction $\phi$ occupied by polyion (Fig. 5). The osmotic lowering due to fixed charges of the protein is significant (10-25\%) and, in conjunction with the intrinsic nonideality of adrenalin-ATP mixtures, accounts reasonably for the apparent nonideality of the intragranular matrix.

It is interesting to note that the osmotic lowering produced by the soluble protein would increase markedly if nucleotides were bound to cationic sidechains of chromogranin. The net fixed negative charge would then increase, requiring an increased fraction of the mobile catecholamine to act as counterion, thus leading to a substantial decrease in $g^{\mathrm{c}}$. The stoichiometry of bound and counterion components is shown in Fig. 5. If, for example, $15 \%$ of the endogenous nucleotide were site-localized by cationic sidechains of chro- 

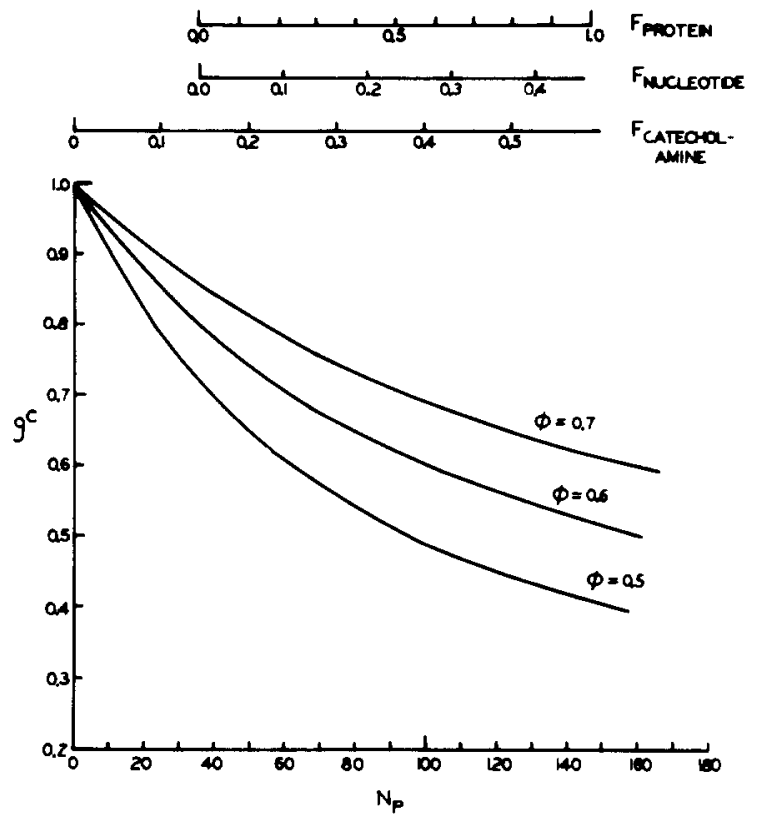

Fig. 5. Theoretical lowering of the osmotic coefficient, $g^{\mathrm{c}}$, in a polyion-counterion solution. $N_{\mathrm{p}}$ is the net charge per 77000 unit of the polyion; $\phi$ is the fraction of the solution volume occupied by the polyion. $F_{\text {catecholamine }}$ and $F_{\text {nucleotide }}$ are the fractions of the total catecholamine (nucleotide) bound to the protein. $F_{\text {protein }}$ is the fraction of cationic amino acid residues neutralized by the bound nucleotide.

mogranin, this would increase the net number of fixed charges to about $-70 e^{-}$per $77000 \mathrm{Da}$ unit, and would approximately double the osmotic lowering due to polyion-counterion interactions.

The results in Fig. 5 show that the soluble protein, simply through its polyion properties, produces significant osmostic lowering of the chromaffin granule aqueous phase. Aggregation of chromogranin with even a minor fraction of the endogenous nucleotide will further increase the nonideality. Whether such aggregation occurs is difficult to determine, although the available evidence clearly indicates that the binding, if present, is very labile and that bound nucleotides comprise no more than a small fraction of the total endogenous pool. Evidence favoring weak interactions of this type includes the differential lowering of the rotational mobility of ATP relative to that of epinephrine in the presence of chromogranins. It has been reported that the undialysable chromaffin granule lysate exhibits substantial binding capacity both for nucleotides and catecholamines, probably through ion-exchange type interactions, although isolated chromogranins appear to have little affinity for ATP [17-20]. Irrespective of whether specific nucleotide-protein binding occurs, chromogranin will contribute to the osmotic lowering of the chromaffin granule aqueous phase. It seems likely at present that the intrinsic nonideality of binary catecholamine-ATP solution is the major source of osmotic stabilization and that chromogranin plays a smaller $(20-40 \%)$ role.

\section{Acknowledgement}

Partial support of this research was obtained in the form of a grant from the Michigan Heart Association.

\section{References}

1 Sen, R., Sharp, R.R., Domino, L.E. and Domino, E.F. (1979) Biochim. Biophys. Acta 587, 75-88

2 Weiner, N. and Jardetsky, O. (1964) Naunyn Schmiedeberg's Arch. Exp. Pathol. Pharmakol. 248, 308-318

3 Berneis, K.H., Pletscher, A. and Da Prada, M. (1969) Nature 244, 281-283

4 Helle, K.B. and Serck-Hanssen, G. (1975) Mol. Cell Biochem. $6,127-146$

5 Berneis, K.H., Pletscher, A. and Da Prada, M. (1970) Br. J. Pharmacol. 39, 382-389

6 Smythies, J.R., Antun, F., Yank, G. and Yorke, C. (1971) Nature 231, 185-188

7 Sharp, R.R. and Richards, E.P. (1977) Biochim. Biophys. Acta 497, 260-271

8 Steffen, H., Da Prada, M. and Pletscher, A. (1974) Biochim. Biophys. Acta 338, 561-571

9 Morris, S.J., Schultens, H.A. and Schober, R. (1977) Biophys. J. 20, 33-48

10 Maynert, E.W., Moon, B.H. and Pai, V.S. (1971) Mol. Pharmacol. 8, 88-94

11 Pai, V.S. and Maynert, E.W. (1972) Mol. Pharmacol. 8, $82-87$

12 Muro, I., Morishima, I. and Yonizawa, T. (1971) Chem. Biol. Interact. 3, 213-224

13 Tuck, L.D. and Baker, J.K. (1973) Chem. Biol. Interact. 7, 355-366

14 Daniels, A., Korda, A. Tanswell, P., Williams, A. and Williams, R.J.P. (1974) Proc. Roy. Soc. Lond. B187, 353-361

15 Daniels, A.J., Williams, R.J.P. and Wright, P.E. (1978) Neuroscience 3, 573-585

16 Granot, J. (1978) J. Am. Chem. Soc. 100, 1539-1548

17 Slotkin, T.A. and Kirshner, N. (1971) Mol. Pharmacol. 7, 581-592

18 Smith, W.J. and Kirshner, N. (1967) Mol. Pharmacol. 3, 52-62 
19 Helle, K.B. (1966) Mol. Pharmacol. 2, 298-310

20 Uvnas, B. and Aborg. C. (1977) Acta Physiol. Scand. 109, 345-354

21 Seeley, P.J., Njus, D., Radda, G.K. and Sehr, P.A. (1977) Biochemistry 16, 972-977

22 Johnson, R.G. and Scarpa, A. (1976) J. Biol. Chem. 25I, 2189-2191

23 Kirshner, N. (1962) J. Biol. Chem. 237, 2311-2317

24 Lowry, O.H., Rosebrough, N.J., Farr, A.L. and Randall, P. (1951) J. Biol. Chem. 193, 265-274

25 Weil-Malherbe, H. (1973) Methods of Biochemical Analysis (Glick, D., ed.), Suppl. Vol. 21, pp. 119-152, Interscience, New York

26 Farrar, T.C. and Becker, E.D. (1971) Pulse and Fourier Transform NMR, Academic Press, New York

27 Carrington, A. and McLachlan, A.D. (1967) Introduction to Magnetic Resonance, Harper and Rowe, New York

28 Abragam, A. (1961) The Principles of Magnetic Resonance, Oxford University Press, New York

29 Doddrell, D., Glushko, V. and Allerhand, A. (1972) J. Chem. Phys. 56, 3683-3689

30 Lam, Y., Kuntz, G.P.P. and Kotowycz, G. (1973) J. Am. Chem. Soc. 96, 1834-1839

31 Weser, U., Strobel, G. and Voelter, W. (1974) FEBS Lett. $41,243-247$

32 Berthod, H. and Pullman, B. (1972) in the Purines: Theory and Experiment (Bergmann, E.D. and Pullman, B., eds.) pp. 30-42, Academic Press, New York

33 Berneis, K.H., Da Prada, M. and Pletscher, A. (1970) Biochim. Biophys. Acta 215, 547-549
34 Berneis, K.H., Da Prada, M. and Pletscher, A. (1974) Nature 248, 604-605

35 Njus, D., Sehr, P.A., Radda, G.K., Ritchie, G.A. and Seeley, J.P. (1978) Biochemistry 17, 4337-4343

36 Granot, J. and Rosenheck, K. (1978) FEBS Lett. 95, 45-48

37 Granot, J. and Fiat, D. (1977) J. Am. Chem. Soc. 99, 4963-4969

38 Komoroski, R.A. (1973) Ph.D. Thesis, Indiana University, University Microfilms, Ann Arbor, MI, U.S.A. Order No. 74-387

39 Sen, R. and Sharp, R.R. (1981) Biochem. J. 195, 329-332

40 Winkler, H. (1976) Neuroscience 1, 65-80

41 Phillips, J.H., Allison, Y.P. and Morris, S.J. (1977) Neuroscience 2, 147-152

42 Winkler, H. and Westhead, E. (1980) Neuroscience S, $1803-1823$

43 Sen, R. (1980) Ph.D. Thesis, The University of Michigan. University Microfilms, Ann Arbor, MI, U.S.A., Order No. 80-25769

44 Njus, D. and Radda, G.K. (1978) Biochim. Biophys. Acta 463, 219-244

45 Granot, J. and Rosenheck, K. (1978) FEBS Lett. 95, 45-48

46 Bovey, F.A. (1972) High Resolution NMR of Macrolecules, Academic Press, New York

47 Sharp, R.R. and Richards, E.P. (1977) Biochim. Biophys. Acta 497, 14-28

48 Smith, A.D. and Winkler, H. (1967) Biochem. J. 103, 483492

49 Oosawa, F. (1971) Polyelectrolytes, Marcel-Dekker, New York 